\title{
SHEFFER POLYNOMIALS AND APPROXIMATION OPERATORS
}

\author{
EMIL C. POPA
}

\begin{abstract}
In this paper we are studying the sequence of linear positive operators $\left(P_{n}^{(Q, S)}\right)$ defined in (2). Using the Bohman-Korovkin uniform convergence criterion we are proving that the sequence $\left(P_{n}^{(Q, S)}\right)$ converges uniformly to the identity operator.

In addition we give some estimates. Finally we consider two examples $\left(P_{n}^{(A, S)}\right)$ and $\left(P_{n}^{(\nabla, S)}\right)$ defined in (25), (27).
\end{abstract}

\section{Introduction}

Let $\Pi$ be the algebra of all polynomials in one variable, with real coefficients and let $Q: \Pi \rightarrow \Pi$ be a delta operator with basic polynomial set $\left(p_{n}\right)$.

Operators $L_{n}: C[0,1] \rightarrow C[0,1], n=0,1,2, \ldots$ of the form

$$
\left(L_{n} f\right)(x)=\frac{1}{p_{n}(1)} \sum_{k=0}^{n}\left(\begin{array}{l}
n \\
k
\end{array}\right) p_{k}(x) p_{n-k}(1-x) f\left(\frac{k}{n}\right)
$$

have been studied in [1]-[2], [4]-[8], [10]-[11].

Next we consider a Sheffer set relative to $Q$, namely $s_{0}(x)=c \neq 0$ and $Q s_{n}(x)=$ $n s_{n-1}(x)$.

Let $S$ be an invertible shift invariant operator such that $S s_{n}(x)=p_{n}(x)$.

Definition. $(Q, S)$ belongs to the class $W$ if the following conditions are satisfied

i) $p_{n}^{\prime} \geq 0, s_{n}(0) \geq 0, s_{0}(x)=1, s_{n}(1) \neq 0$ for $n=1,2,3, \ldots$.

ii) $\lim _{n \rightarrow \infty} \frac{\sum_{i=2}^{n}\left(\begin{array}{c}n-2 \\ i-2\end{array}\right) s_{n-i}(0)\left(Q^{\prime-2} p_{i-2}\right)(1)}{s_{n}(1)}=1$.

where $Q^{\prime}$ is the Pincherle derivative of the operator $Q$.

The identity

$$
s_{n}(x+y)=\sum_{k=0}^{n}\left(\begin{array}{l}
n \\
k
\end{array}\right) p_{k}(x) s_{n-k}(y)
$$

Received January 29, 2001.

2000 Mathematics Subject Classification. 41A36, 05A40.

Key words and phrases. Delta operator, Pincherle derivative, Sheffer polynomials, Abel operator, backward difference operator, approximation operators. 
which characterizes the Sheffer polynomials, suggest us to consider the sequence of linear polynomial operators $P_{n}^{(Q, S)}: C[0,1] \rightarrow C[0,1]$ defined by

$$
\left(P_{n}^{(Q, S)} f\right)(x)=\frac{1}{s_{n}(1)} \sum_{k=0}^{n}\left(\begin{array}{l}
n \\
k
\end{array}\right) p_{k}(x) s_{n-k}(1-x) f\left(\frac{k}{n}\right)
$$

where $(Q, S) \in W, f \in C[0,1]$.

For example if $Q=D E^{-\beta}, p_{n}(x)=x(x+n \beta)^{n-1}, s_{n}(x)=(x+n \beta)^{n}$ we obtain the Cheney-Sharma operator (1964, see [2])

$$
\left(S_{n} f\right)(x)=\sum_{k=0}^{n}\left(\begin{array}{l}
n \\
k
\end{array}\right) \frac{x(x+k \beta)^{k-1}(1-x+\overline{n-k} \beta)^{n-k}}{(1+n \beta)^{n}} f\left(\frac{k}{n}\right)
$$

where $E^{-\beta}$ is the shift operator and $D$ the derivative.

Lemma 1. If $(Q, S) \in W$ then $P_{n}^{(Q, S)}, n=1,2,3, \ldots$ are positive operators.

Proof. From the identities (see [9])

$$
\begin{aligned}
& p_{n}(x)=x \sum_{k=0}^{n-1}\left(\begin{array}{c}
n-1 \\
k
\end{array}\right) p_{n-k-1}(x) p_{k+1}^{\prime}(0) \\
& s_{n}(x)=\sum_{k=0}^{n}\left(\begin{array}{l}
n \\
k
\end{array}\right) s_{k}(0) p_{n-k}(x)
\end{aligned}
$$

on obtain $p_{n}(x) \geq 0, s_{n}(x) \geq 0, n=1,2, \ldots$ for every $x \in[0,1]$.

Lemma 2. If $p_{n}^{\prime}(0) \geq 0$ for $n=1,2, \ldots$, then

$$
0<\left(Q^{\prime-2} p_{n-2}\right)(1) \leq p_{n}(1), \quad n=2,3, \ldots
$$

Proof. According to theorem 9 from [4], and lemma 1 we have

$$
\frac{p_{n-1}(x)}{x} p_{1}^{\prime}(0) \leq\left(Q^{\prime-2} p_{n-2}\right)(x) \leq \frac{p_{n}(x)}{x^{2}}, \quad x>0, n=2,3, \ldots
$$

For $x=1$ this gives

$$
0<\left(Q^{\prime-2} p_{n-2}\right)(1) \leq p_{n}(1) \quad \text { for } \quad n=2,3, \ldots
$$

\section{Results}

If $m$ is a natural number, let us denote

$$
S_{m}(x, y, n)=\sum_{k=0}^{n}\left(\begin{array}{l}
n \\
k
\end{array}\right) p_{k}(x) s_{n-k}(y)\left(\frac{k}{n}\right)^{m}
$$


and let $P$ be the linear operator defined by $P=x Q^{\prime-1}$.

Using the method from [4] or [6] and the identity

$$
s_{n}(x+y)=\sum_{k=0}^{n}\left(\begin{array}{l}
n \\
k
\end{array}\right) p_{k}(x) s_{n-k}(y)
$$

we obtain

$$
S_{m}(x, y, n)=\frac{1}{n^{m}} \sum_{k=0}^{m} k !\left(\begin{array}{l}
n \\
k
\end{array}\right) S(m, k) P^{k} E^{y} s_{n-k}(x)
$$

where $S(m, k)$ are the Stirling numbers of second kind and $E^{y}$ is the shift operator $\left(E^{y} f\right)(x)=f(x+y)$.

Taking into account that

$$
\begin{aligned}
P E^{y} p_{n-1}(x) & =\frac{x}{x+y} p_{n}(x+y) \\
s_{n-1}(x) & =\sum_{k=0}^{n-1}\left(\begin{array}{c}
n-1 \\
k
\end{array}\right) s_{n-k-1}(0) p_{k}(x)
\end{aligned}
$$

(see [6]) we obtain

$$
P E^{y} s_{n-1}(x)=\frac{x}{x+y} \sum_{i=1}^{n}\left(\begin{array}{c}
n-1 \\
i-1
\end{array}\right) s_{n-i}(0) p_{i}(x+y)
$$

Now using the fact that

$$
P^{2} E^{y} p_{n-2}(x)=\frac{x}{x+y} p_{n}(x+y)-x y Q^{\prime-2} p_{n-2}(x+y)
$$

(see $[6])$ and

$$
s_{n-2}(x)=\sum_{k=0}^{n-2}\left(\begin{array}{c}
n-2 \\
k
\end{array}\right) s_{n-k-2}(0) p_{k}(x)
$$

we obtain

$$
\begin{gathered}
P^{2} E^{y} s_{n-2}(x)=\frac{x}{x+y} \sum_{i=2}^{n}\left(\begin{array}{c}
n-2 \\
i-2
\end{array}\right) s_{n-i}(0) p_{i}(x+y) \\
-x y \sum_{i=2}^{n}\left(\begin{array}{c}
n-2 \\
i-2
\end{array}\right) s_{n-i}(0) Q^{\prime-2} p_{i-2}(x+y)
\end{gathered}
$$

Next we have

$$
\begin{aligned}
& S_{0}(x, y, n)=s_{n}(x+y) \\
& S_{1}(x, y, n)=\frac{x}{x+y} \sum_{i=1}^{n}\left(\begin{array}{c}
n-1 \\
i-1
\end{array}\right) s_{n-i}(0) p_{i}(x+y)
\end{aligned}
$$




$$
\begin{aligned}
S_{2}(x, y, n)= & \frac{1}{n} \frac{x}{x+y} \sum_{i=1}^{n}\left(\begin{array}{c}
n-1 \\
i-1
\end{array}\right) s_{n-i}(0) p_{i}(x+y)+\frac{n-1}{n} \frac{x}{x+y} \sum_{i=2}^{n}\left(\begin{array}{c}
n-2 \\
i-2
\end{array}\right) s_{n-i}(0) p_{i}(x+y) \\
& -\frac{n-1}{n} x y \sum_{i=2}^{n}\left(\begin{array}{c}
n-2 \\
i-2
\end{array}\right) s_{n-i}(0)\left(Q^{\prime-2} p_{i-2}\right)(x+y)
\end{aligned}
$$

Hence

$$
\begin{aligned}
\left(P_{n}^{(Q, S)} e_{0}\right)(x)= & e_{0} \\
\left(P_{n}^{(Q, S)} e_{1}\right)(x)= & \frac{\sum_{i=1}^{n}\left(\begin{array}{c}
n-1 \\
i-1
\end{array}\right) s_{n-i}(0) p_{i}(1)}{s_{n}(1)} x \\
\left(P_{n}^{(Q, S)} e_{2}\right)(x)= & \frac{1}{n} \frac{\sum_{i=1}^{n}\left(\begin{array}{c}
n-1 \\
i-1
\end{array}\right) s_{n-i}(0) p_{i}(1)}{s_{n}(1)} x+\frac{n-1}{n} \frac{\sum_{i=2}^{n}\left(\begin{array}{c}
n-2 \\
i-2
\end{array}\right) s_{n-i}(0) p_{i}(1)}{s_{n}(1)} x \\
& -\frac{n-1}{n} \frac{\sum_{i=2}^{n}\left(\begin{array}{c}
n-2 \\
i-2
\end{array}\right) s_{n-i}(0)\left(Q^{\prime-2} p_{i-2}\right)(1)}{s_{n}(1)} x(1-x)
\end{aligned}
$$

where $e_{k}(x)=x^{k}, k=0,1,2, \ldots$.

Let us denote

$$
\begin{aligned}
a_{n} & =\frac{\sum_{i=1}^{n}\left(\begin{array}{c}
n-1 \\
i-1
\end{array}\right) s_{n-i}(0) p_{i}(1)}{s_{n}(1)} \\
b_{n} & =\frac{\sum_{i=2}^{n}\left(\begin{array}{c}
n-2 \\
i-2
\end{array}\right) s_{n-i}(0) p_{i}(1)}{s_{n}(1)} \\
c_{n} & =\frac{s_{n-1}(0) p_{1}(1)+\sum_{i=2}^{n}\left(\begin{array}{c}
n-2 \\
i-2
\end{array}\right) s_{n-i}(0) p_{i}(1)}{s_{n}(1)} \\
d_{n} & =\frac{\sum_{i=2}^{n}\left(\begin{array}{c}
n-2 \\
i-2
\end{array}\right) s_{n-i}(0)\left(Q^{\prime-2} p_{i-2}\right)(1)}{s_{n}(1)}
\end{aligned}
$$

Therefore

$$
\begin{gathered}
\left(P_{n}^{(Q, S)} e_{1}\right)(x)=a_{n} x \\
\left(P_{n}^{(Q, S)} e_{2}\right)(x)=b_{n} x^{2}+\frac{1}{n} c_{n} x+\left(x-x^{2}\right)\left(b_{n}-\frac{n-1}{n} d_{n}\right)
\end{gathered}
$$

Taking into account that

$$
\left(\begin{array}{c}
n-2 \\
i-2
\end{array}\right) \leq\left(\begin{array}{c}
n-1 \\
i-1
\end{array}\right) \leq\left(\begin{array}{c}
n \\
i
\end{array}\right), \quad(Q, S) \in W
$$

and using lemma 2, we obtain

$$
d_{n} \leq \frac{\sum_{i=2}^{n}\left(\begin{array}{c}
n-2 \\
i-2
\end{array}\right) s_{n-i}(0) p_{i}(1)}{s_{n}(1)}=b_{n} \leq \frac{\sum_{i=2}^{n}\left(\begin{array}{c}
n-1 \\
i-1
\end{array}\right) s_{n-i}(0) p_{i}(1)}{s_{n}(1)} \leq a_{n} \leq \frac{\sum_{i=1}^{n}\left(\begin{array}{c}
n \\
i
\end{array}\right) s_{n-i}(0) p_{i}(1)}{s_{n}(1)} \leq 1
$$


whence

$$
\lim _{n \rightarrow \infty} a_{n}=\lim _{n \rightarrow \infty} b_{n}=1 .
$$

Now using $\frac{n-i}{i-1}\left(\begin{array}{c}n-2 \\ i-2\end{array}\right) \leq\left(\begin{array}{c}n \\ i\end{array}\right)$ for $i=2,3, \ldots, n$, we have

$$
0 \leq c_{n} \leq \frac{n s_{n-1}(0) p_{1}(1)+\sum_{i=2}^{n}\left(\begin{array}{c}
n \\
i
\end{array}\right) s_{n-i}(0) p_{i}(1)}{s_{n}(1)}=\frac{\sum_{i=1}^{n}\left(\begin{array}{c}
n \\
i
\end{array}\right) s_{n-i}(0) p_{i}(1)}{s_{n}(1)} \leq 1
$$

Hence

$$
\lim _{n \rightarrow \infty}\left(\frac{1}{n} c_{n}\right)=0
$$

Theorem 1. If $(Q, S) \in W$ and $f \in C[0,1]$, then

$$
\lim _{n \rightarrow \infty}\left\|f-P_{n}^{(Q, S)} f\right\|=0
$$

where $\|f\|=\max _{t \in[0,1]}|f(t)|$.

Proof. According to the Bohman-Korovkin theorem it is sufficient to show that

$$
\lim _{n \rightarrow \infty}\left\|e_{k}-P_{n}^{(Q, S)} e_{k}\right\|=0, \quad k=0,1,2 .
$$

In our case, from (10), (13), (14)

$$
\begin{gathered}
\left\|e_{0}-P_{n}^{(Q, S)} e_{0}\right\|=0 \\
\left\|e_{1}-P_{n}^{(Q, S)} e_{1}\right\|=\left\|x-a_{n} x\right\|=1-a_{n}
\end{gathered}
$$

and $\lim _{n \rightarrow \infty}\left\|e_{1}-P_{n}^{(Q, S)} e_{1}\right\|=0$.

Finally

$$
\begin{aligned}
\left\|e_{2}-P_{n}^{(Q, S)} e_{2}\right\| & =\left\|\left(1-b_{n}\right) x^{2}-\frac{1}{n} c_{n} x-\left(x-x^{2}\right)\left(b_{n}-\frac{n-1}{n} d_{n}\right)\right\| \\
& \leq\left(1-b_{n}\right)+\frac{1}{n} c_{n}+\frac{1}{4}\left(b_{n}-\frac{n-1}{n} d_{n}\right)
\end{aligned}
$$

where $1-\frac{1}{n} \leq 1 \leq \frac{b_{n}}{d_{n}}$.

Therefore

$$
\lim _{n \rightarrow \infty}\left\|e_{2}-P_{n}^{(Q, S)} e_{2}\right\|=0
$$

Theorem 2. Let $\left(P_{n}^{(Q, S)}\right),(Q, S) \in W$ be the sequence of linear positive operators defined in (2). If $f \in C^{2}[0,1], m_{f}=\min _{x \in[0,1]} f^{\prime \prime}(x), M_{f}=\max _{x \in[0,1]} f^{\prime \prime}(x)$, then for $x \in[0,1]$

$$
\frac{1}{2} m_{f} \theta_{n}(x) \leq\left(P_{n}^{(Q, S)} f\right)(x)-f\left(a_{n} x\right) \leq \frac{1}{2} M_{f} \theta_{n}(x)
$$


where $\theta_{n}=\left(P_{n}^{(Q, S)} e_{2}\right)(x)-a_{n}^{2} x^{2}$.

Proof. If $h \in C[0,1]$ is convex on $I, c_{1}, c_{1}, \ldots, c_{n}$ are non-negative numbers with $c_{0}+c_{1}+\cdots+c_{n}=1$, then for every system of points $x_{0}, x_{1}, \ldots, x_{n}$ from $[0,1]$

$$
h\left(\sum_{k=0}^{n} c_{k} x_{k}\right) \leq \sum_{k=0}^{n} c_{k} h\left(x_{k}\right) .
$$

Let us consider

$$
\begin{gathered}
c_{k}=\frac{1}{s_{n}(1)}\left(\begin{array}{l}
n \\
k
\end{array}\right) p_{k}(x) s_{n-k}(1-x) \\
x_{k}=\frac{k}{n}
\end{gathered}
$$

$x$ being arbitrary in $[0,1]$. Then

$$
\sum_{k=0}^{n} c_{k} x_{k}=\left(P_{n}^{(Q, S)} e_{1}\right)(x)=a_{n} x
$$

and hence

$$
h\left(a_{n} x\right) \leq\left(P_{n}^{(Q, S)} h\right)(x)
$$

Now we observe that:

$$
h_{1}(x)=\frac{1}{2} M_{f} x^{2}-f(x) ; \quad h_{2}(x)=f(x)-\frac{1}{2} m_{f} x^{2}
$$

are convex on $[0,1]$.

Hence

$$
\begin{aligned}
& h_{1}\left(a_{n} x\right) \leq\left(P_{n}^{(Q, S)} h_{1}\right)(x) \\
& h_{2}\left(a_{n} x\right) \leq\left(P_{n}^{(Q, S)} h_{2}\right)(x)
\end{aligned}
$$

and

$$
\begin{aligned}
& \left(P_{n}^{(Q, S)} h_{1}\right)(x)=\frac{1}{2} M_{f}\left(P_{n}^{(Q, S)} e_{2}\right)(x)-\left(P_{n}^{(Q, S)} f\right)(x) \\
& \left(P_{n}^{(Q, S)} h_{2}\right)(x)=\left(P_{n}^{(Q, S)} f\right)(x)-\frac{1}{2} m_{f}\left(P_{n}^{(Q, S)} e_{2}\right)(x)
\end{aligned}
$$

Now using (17)

$$
\begin{aligned}
\frac{1}{2} M_{f} a_{n}^{2} x^{2}-f\left(a_{n} x\right) & \leq \frac{1}{2} M_{f}\left(P_{n}^{(Q, S)} e_{2}\right)(x)-\left(P_{n}^{(Q, S)} f\right)(x) \\
f\left(a_{n} x\right)-\frac{1}{2} m_{f} a_{n}^{2} x^{2} & \leq\left(P_{n}^{(Q, S)} f\right)(x)-\frac{1}{2} m_{f}\left(P_{n}^{(Q, S)} e_{2}\right)(x)
\end{aligned}
$$

Finally

$$
\frac{1}{2} m_{f}\left[\left(P_{n}^{(Q, S)} e_{2}\right)(x)-a_{n}^{2} x^{2}\right] \leq\left(P_{n}^{(Q, S)} f\right)(x)-f\left(a_{n} x\right) \leq \frac{1}{2} M_{f}\left[\left(P_{n}^{(Q, S)} e_{2}\right)(x)-a_{n}^{2} x^{2}\right]
$$


Theorem 3. Let $(Q, S) \in W, f \in C[0,1]$ and denote by $w(f ; \delta)$ the modulus of continuity of the function $f$. If $x \in[0,1]$, then

$$
\begin{aligned}
\left|f(x)-\left(P_{n}^{(Q, S)} f\right)(x)\right| & \leq 2 w\left(f ; \sqrt{A_{n}}\right) \\
\left\|f-P_{n}^{(Q, S)} f\right\| & \leq 2 w\left(f ; \sqrt{B_{n}}\right)
\end{aligned}
$$

where

$$
\begin{aligned}
& A_{n}=\left(b_{n}-2 a_{n}+1\right) x^{2}+\frac{1}{n} c_{n} x+\left(x-x^{2}\right)\left(b_{n}-\frac{n-1}{n} d_{n}\right) \\
& B_{n}=\left(b_{n}-2 a_{n}+1\right)+\frac{1}{n} c_{n}+\frac{1}{4}\left(b_{n}-\frac{n-1}{n} d_{n}\right)
\end{aligned}
$$

Proof. If $L: C[0,1] \rightarrow C[0,1]$ is a linear positive operator, then (see for instance theorem 4.2 and 4.5 from [3])

$$
\begin{aligned}
\left|f(x)-\left(L_{n} f\right)(x)\right| & \leq 2 w\left(f ; \sqrt{\left(L \Omega_{2}\right)(x)}\right. \\
\|f-L f\| & \leq \inf _{m=1,2, \ldots}\left\{1+\delta^{-m}\left\|L \Omega_{m}\right\|\right\} w(f, \delta)
\end{aligned}
$$

where $\delta>0, \Omega_{j}(t, x)=\Omega_{j}(t)=|t-x|^{j}$.

We have

$$
\left(P_{n}^{(Q, S)} \Omega_{2}\right)(x)=\left(1-2 a_{n}+b_{n}\right) x^{2}+\frac{1}{n} c_{n} x+\left(x-x^{2}\right)\left(b_{n}-\frac{n-1}{n} d_{n}\right)
$$

We observe that

$1-2 a_{n}+b_{n}=s_{n}(0)+(n-2) s_{n-1}(0) p_{1}(1)+\sum_{i=2}^{n}\left[\left(\begin{array}{c}n-2 \\ i-2\end{array}\right)+\left(\begin{array}{c}n \\ i\end{array}\right)-2\left(\begin{array}{c}n-1 \\ i-1\end{array}\right)\right] s_{n-i}(0) p_{i}(1) \geq 0$

for $n=2,3, \ldots$.

Therefore

$$
\left(P_{n}^{(Q, S)} \Omega_{2}\right)(x) \geq 0 \quad \text { for } \quad x \in[0,1]
$$

and

$$
\left|f(x)-\left(P_{n}^{(Q, S)} f\right)(x)\right| \leq 2 w\left(f ; \sqrt{A_{n}}\right) .
$$

For $m=2$ in (19) we obtain

$$
\left\|f-P_{n}^{(Q, S)} f\right\| \leq 2 w\left(f ; \sqrt{B_{n}}\right) .
$$

\section{Examples}

Let us consider the following examples 
I. The Abel operator $A=D E^{-\alpha}$ is a delta operator with basic polynomials

$$
p_{n}^{\alpha}=x(x+n \alpha)^{n-1}
$$

and we consider that

$$
\alpha=\alpha(n)>0, \quad \lim _{n \rightarrow \infty} n^{3} \alpha(n)=0
$$

Let $\left(s_{n}^{\alpha}\right)$ be the Sheffer set

$$
s_{n}^{\alpha}=(x+\overline{n-1} \alpha)^{n}, \quad s_{0}(x)=1
$$

namely $A s_{n}=n s_{n-1}, n=1,2,3, \ldots$

We have

i) $\left[p_{n}^{\alpha}\right]^{\prime}(0)=(n \alpha)^{n-1}>0, s_{n}(0)=(n-1)^{n} \alpha^{n} \geq 0, s_{n}^{\alpha}(1)=(1+\overline{n-1} \alpha)^{n} \neq 0$.

ii) According to theorem 9 (from [4]) we have

$$
\frac{p_{n-1}^{\alpha}(1)}{p_{n}^{\alpha}(1)}\left[p_{1}^{\alpha}\right]^{\prime}(0) \leq \frac{\left(Q^{\prime-2} p_{n-2}^{\alpha}\right)(1)}{p_{n}^{\alpha}(1)} \leq 1, \quad n=2,3, \ldots
$$

From (22)

$$
\lim _{n \rightarrow \infty} \alpha(n)=\lim _{n \rightarrow \infty} n \alpha(n)=\ln n^{2} \alpha(n)=0
$$

Hence

$$
\lim _{n \rightarrow \infty} \frac{p_{n-1}^{\alpha}(1)}{p_{n}^{\alpha}(1)}=\lim _{n \rightarrow \infty} \frac{(1+\overline{n-1} \alpha)^{n-1}}{(1+n \alpha)^{n-1}}=1
$$

and using $\left(p_{1}^{\alpha}\right)^{\prime}(0)=1$ we have

$$
\lim _{n \rightarrow \infty} \frac{\left(Q^{\prime-2} p_{n-2}^{\alpha}\right)(1)}{p_{n}^{\alpha}(1)}=1
$$

Now

$$
\lim _{n \rightarrow \infty} \frac{p_{n}^{\alpha}(1)}{s_{n}^{\alpha}(1)}=\lim _{n \rightarrow \infty} \frac{(1+n \alpha)^{n-1}}{(1+\overline{n-1} \alpha)^{n}}=1
$$

From (23), (24) we obtain

$$
\lim _{n \rightarrow \infty} \frac{\left(Q^{\prime-2} p_{n-2}^{\alpha}\right)(1)}{s_{n}^{\alpha}(1)}=1 .
$$

Now using (15) we observe that

$$
\frac{\left(Q^{\prime-2} p_{n-2}^{\alpha}\right)(1)}{s_{n}^{\alpha}} \leq d_{n} \leq 1
$$

namely $\lim _{n \rightarrow \infty} d_{n}=1$.

Therefore $(A, S) \in W$ and the sequence of linear polinomial operator

$$
P_{n}^{(A, S)}: C[0,1] \rightarrow C[0,1]
$$


defined by

$$
\left(P_{n}^{(A, S)} f\right)(x)=\frac{1}{(1+\overline{n-1} \alpha)^{n}} \sum_{k=0}^{n}\left(\begin{array}{l}
n \\
k
\end{array}\right) x(x+k \alpha)^{k-1}(1-x+\overline{n-k-1} \alpha)^{n-k} f\left(\frac{k}{n}\right)
$$

with $\alpha=\alpha(n)>0, \ln n^{3} \alpha(n)=0$, verify

$$
\lim _{n \rightarrow \infty}\left\|f-P_{n}^{(A, S)} f\right\|=0
$$

for every $C[0,1]$, where

$$
\|f\|=\max _{t \in[0,1]}|f(t)| .
$$

II. Backward difference operator

$$
\nabla=\frac{1}{\alpha}\left(I-E^{-\alpha}\right)
$$

where $\alpha=\alpha(n)>0, \lim _{n \rightarrow \infty} \alpha(n)=0$, with the basic sequence

$$
p_{n}^{\alpha}(x)=x(x+\alpha) \cdots(x+\overline{n-1} \alpha), \quad p_{0}(x)=1 .
$$

Let $\left(s_{n}^{\alpha}\right)$ be a Sheffer set relative to $\nabla$ defined by

$$
s_{n}^{\alpha}(x)=x(x+\alpha) \cdots(x+\overline{n-2} \alpha)(x+\overline{n-1} \alpha+n), \quad s_{0}(x)=1
$$

We have

i) $\left[p_{n}^{\alpha}\right]^{\prime}(0)=(n-1) ! \alpha^{n-1}>0, s_{n}(0) \geq 0, n=1,2, \ldots, s_{n}^{\alpha}(1) \neq 0$;

ii) We observe that

$$
\frac{\left(\nabla^{\prime-2} p_{n-2}^{\alpha}\right)(1)+(n-2)\left(\nabla^{\prime-2} p_{n-3}^{\alpha}\right)(1)}{s_{n}^{\alpha}(1)} \leq d_{n} \leq 1, \quad n=3,4, \ldots
$$

But

$$
\begin{aligned}
& \left(\nabla^{\prime-2} p_{n-2}^{\alpha}\right)(1)=(1+2 \alpha)(1+3 \alpha) \cdots(1+\overline{n-1} \alpha) \\
& \left(\nabla^{\prime-2} p_{n-3}^{\alpha}\right)(1)=(1+2 \alpha)(1+3 \alpha) \cdots(1+\overline{n-2} \alpha),
\end{aligned}
$$

whence

$$
\lim _{n \rightarrow \infty} \frac{\left(\nabla^{\prime-2} p_{n-2}^{2}\right)(1)+(n-2)\left(\nabla^{\prime-2} p_{n-3}^{\alpha}\right)(1)}{s_{n}^{\alpha}(1)}=\lim _{n \rightarrow \infty} \frac{1+\overline{n-1} \alpha+n-2}{(1+\alpha)(1+\overline{n-1} \alpha+n)}=1
$$

and

$$
\lim _{n \rightarrow \infty} d_{n}=1
$$


Therefore $(\nabla, S) \in W$ and the sequence of linear polinomial operators

$$
P_{n}^{(\nabla, S)}: C[0,1] \rightarrow C[0,1]
$$

defined by

$$
\left(P_{n}^{(Q, S)} f\right)(x)=\frac{1}{1+\overline{n-1} \alpha+n} \sum_{k=0}^{n} w_{n, k}(x, \alpha) f\left(\frac{k}{n}\right)
$$

where

$$
w_{n, k}(x, \alpha)=\left(\begin{array}{l}
n \\
k
\end{array}\right) \frac{\prod_{i=0}^{k-1}(x+i \alpha) \prod_{i=0}^{n-k-2}(1-x+i \alpha)}{(1+\alpha)(1+2 \alpha) \cdots(1+\overline{n-2} \alpha)}(1-x+\overline{n-k-1} \alpha+n-k)
$$

$\alpha=\alpha(n)>0, \lim _{n \rightarrow \infty} \alpha(n)=0$, verify

$$
\lim _{n \rightarrow \infty}\left\|f-P_{n}^{(\nabla, S)} f\right\|=0, \quad \text { for every } \quad f \in C[0,1] .
$$

Finally we observe that $P_{n}^{(D, I)}$ is the Bernstein operator where $D$ is the derivative operator and $I$ the identity

$$
\left(P_{n}^{(D, I)} f\right)(x)=\left(B_{n} f\right)(x)=\sum_{k=0}^{n}\left(\begin{array}{l}
n \\
k
\end{array}\right) x^{k}(1-x)^{k} f\left(\frac{k}{n}\right)
$$

Operators such as $P_{n}^{(Q, I)}$ had been studied by Brass H. [1], Cheney E. W., Sharma A. [2], Manole C. [6], Moldovan Gr. [7], Mühlbach G. [8], Stancu D. D. [10], [11]

$$
\left(P_{n}^{Q, I} f\right)(x)=\frac{1}{p_{n}(1)} \sum_{k=0}^{n}\left(\begin{array}{l}
n \\
k
\end{array}\right) p_{k}(x) p_{n-k}(1-x) f\left(\frac{k}{n}\right)
$$

For this operators condition ii) of definition becomes

$$
\lim _{n \rightarrow \infty} \frac{\left(Q^{\prime-2} p_{n-2}\right)(1)}{p_{n}(1)}=1 .
$$

\section{References}

[1] Brass, H., Eine verallgemeinerung der Bernsteinschen operatoren, Abhandl. Math. Sem. Hamburg 36(1971), 111-222.

[2] Cheney E. W. and Sharma A., On a generalisation of Bernstein polynomials, Riv. Math. Univ. Parma 5(2)(1964), 77-82.

[3] Lupaş A., Contributions to Approximation Theory through Linear Operators (in Romanian), Doctoral thesis, Cluj-Napoca, 1976.

[4] Lupaş A., On the approximation operators of binomial type, Proc. of International Meeting Approximation Theory, Dortmund, 1998. New Developments in Approximation Theory, Birkhäuser-Verlag-Basel, International Series of Numerical Mathematics, ISNM 132(1999), 175-198. 
[5] Lupaş, L and Lupaş A., Polynomials of binomial type and approximation operators, Studia Univ. Babeş-Bolyai, Mathematica, 32(1987), 61-69.

[6] Manole C., Expansion in Series of Generalized Appell Polynomials with Applications of Approximation Functions (in Romanian), Doctoral thesis, Cluj-Napoca, 1984.

[7] Moldovan Gr., Generalizations of Bernstein Polynomials (in Romanian), Doctoral thesis, Cluj-Napoca, 1971.

[8] Mühlback G., Operatoren von Bernsteinschen typ, J. Approx. Theory 3(1970), 274-292.

[9] Rota G.-C., Kahaner D. and Odlyzko A., On the foundations of combinatorial theory VII, Finite Operator Calculus, J. Math. Anal, Appl. 42(1973), 685-760.

[10] Stancu D. D., Approximation of functions by a new class of linear positive operators, Rev. Roum. Math. Pures et Appl. 13(1968), 1173-1194.

[11] Stancu D. D., Approximation of functions by means of some new classes of positive linear operators, Numerische Methoden der Approximationstheorie, Proc. Conf. Oberwolfach 1971, Birkhäuser-verlag, Basel, ISNM 16(1972), 187-203.

Department of Mathematics, Faculty of Sciences, I. Raţiu, No.5-7, 2400 Sibiu, România.

E-mail: emil.popa@mail.ulbsibiu.ro 